\title{
Inter-hemispheric asymmetry of dependence of the cusp location on dipole tilt during northward IMF conditions
}

\author{
J. Shi ${ }^{1}$, J. Guo ${ }^{2}$, M. Dunlop ${ }^{3}$, T. Zhang ${ }^{4}$, Z. Liu ${ }^{1}$, E. Lucek ${ }^{5}$, A. Fazakerley ${ }^{6}$, H. Rème ${ }^{7}$, and I. Dandouras \\ ${ }^{1}$ State Key Laboratory of Space Weather, CSSAR, Chinese Academy of Sciences, Beijing, China \\ ${ }^{2}$ National Center for Space Weather, China Meteorological Administration, Beijing, China \\ ${ }^{3}$ SSTD, Rutherford Appleton Laboratory, Chilton, Didcot, UK \\ ${ }^{4}$ Space Research Institute, Austrian Academy of Sciences, Graz, Austria \\ ${ }^{5}$ Imperial College London, UK \\ ${ }^{6}$ MSSL, University College London, UK \\ ${ }^{7}$ CESR, Toulouse, France
}

Correspondence to: J. Shi (jkshi@cssar.ac.cn)

Received: 20 May 2011 - Revised: 20 November 2011 - Accepted: 29 November 2011 - Published: 5 January 2012

\begin{abstract}
A data set of the Cluster cusp crossings over a 5year period is studied for the interhemispheric comparison of the dipole tilt angle effect on the latitude of the mid-altitude cusp. The result shows that the dipole tilt angle has a clear control of the cusp latitudinal location. Although, the northern cusp moves $0.054^{\circ}$ ILAT for every $1^{\circ}$ increase in the dipole tilt angle at the mean altitude of $5.2 R_{\mathrm{E}}$, the southern cusp moves $0.051^{\circ}$ ILAT for every $1^{\circ}$ increase in the dipole tilt angle at the mean altitude of $6.6 R_{\mathrm{E}}$. The northern cusp dependence agrees with the trend formed by other observations of different satellites for different altitudes, whereas the southern cusp does not. We therefore suggest that there is an inter-hemispheric difference in the dipole tilt angle dependence of cusp, latitudinal location, which has an impact on other observations of different satellites in different altitudes.
\end{abstract}

Keywords. Ionosphere (Polar ionosphere)

\section{Introduction}

The magnetosheath plasma can directly access the highlatitude ionosphere through the two narrow funnel-shaped cusp regions (Heikkila and Winningham, 1971; Frank, 1971). Accordingly, the two cusps are generally recognized as being key regions for the solar wind-magnetosphereionosphere coupling. Because of the solar wind interaction with the magnetosphere, the two cusps are always changing in their locations and shapes to respond to the time varying solar wind. As the Earth's dipole axis has an angle to its spin axis, the ram pressure of the solar wind, the IMF orientation (i.e. $B_{\mathrm{y}}$, and $B_{\mathrm{x}}$ ) and the $\mathrm{Sq}$ current system around each cusp region in the magnetopause are likely to have some effect on the position of the cusp, the response of the two cusps should be different.

It has been theoretically predicted that the cusp would be several degrees lower in the hemisphere whose dipole axis pointed away from the solar wind ram velocity direction (Choe et al., 1973). Based on the OGO4 data $(700 \mathrm{~km}$ of altitude), Burch (1972) first investigated the effect of dipole tilt angle on the cusp equatorward boundary and found a cusp shifted by approximately $4^{\circ}$ in the invariant latitude (ILAT) over the total yearly range of dipole tilt angles. A survey of the cusp crossings observed by DMSP F7 $(\sim 800 \mathrm{~km}$ of altitude) has shown that the cusp is found to move by $\pm 2^{\circ}$ magnetic latitude (MLAT) from the mean position, which is located between $75.9^{\circ}$ and $76.8^{\circ}$ MLAT, in response to the changing dipole tilt angle (Newell and Meng, 1989).

The cusp has also been studied from high-altitude and mid-altitude satellites. Němev̌ek et al. (2000) found the footprint positions of the cusp-like plasma, which is determined by the observation of the MAGION-4 satellite (altitudes 5 to $15 R_{\mathrm{E}}$ ), to have a substantial latitudinal dependence on the dipole tilt angle with a slope of $0.15^{\circ}$ MLAT per $1^{\circ}$ of dipole tilt angle. This slope increases for observations made in the vicinity of the magnetopause $\left(0.16^{\circ}\right.$ MLAT per $1^{\circ}$ of dipole tilt angle). The observations of the Polar Spacecraft (altitudes 5 to $10 R_{\mathrm{E}}$ ) suggested the same dipole tilt angle effect on the location of the northern cusp, with roughly $0.07^{\circ}$ ILAT for every $1^{\circ}$ of dipole tilt angle at mid-altitude (Zhou et al., 1999). In a recent paper, Pitout et al. (2006) conducted a statistical study of the four years Cluster crossings of the mid-altitude cusp. Their results showed that an increase of $\sim 11^{\circ}$ in dipole tilt angle results in an increase of $1^{\circ}$ in ILAT. 


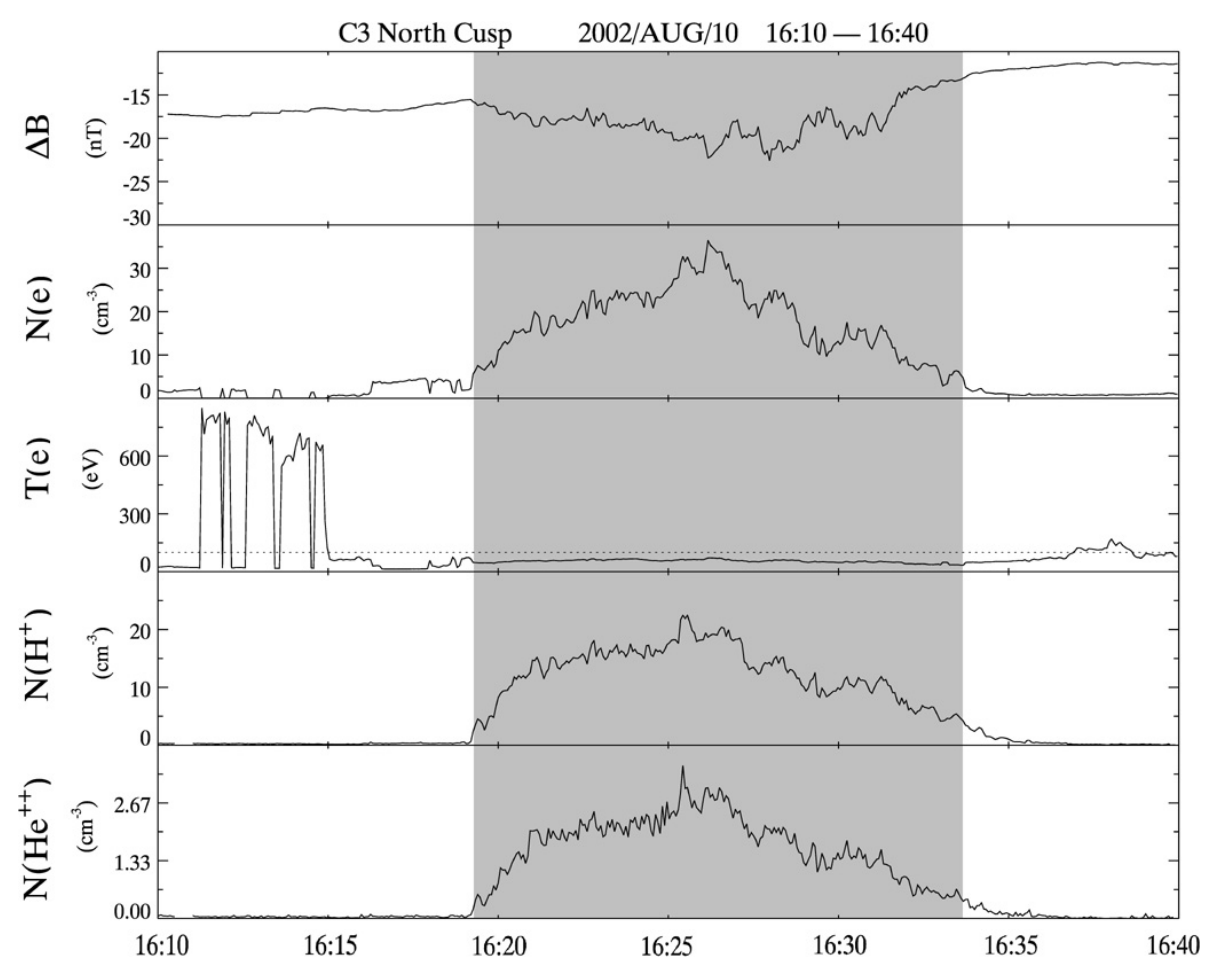

Fig. 1. Example of cusp crossings of Cluster on 10 August 2002: from the upper to bottom panel show the residual of the magnitude of Cluster FGM data with the T96 model, the PEACE electron density and energy, the HIA $\mathrm{H}^{+}$density, and the $\mathrm{He}^{++}$density, respectively. The shadow indicates the polar cusp region based on the criteria.

But they did not take into account the interhemispheric difference in the effect of the dipole tilt angle on the cusp location. Newell et al. (2006) checked the difference between the two hemispheres in the dipole title angle effect by the DMSP satellite observations at the $\sim 800 \mathrm{~km}$ altitude. They gave a slope of $0.043^{\circ}$ cusp latitude per $1^{\circ}$ dipole tile angle by a large number of cases (1857), mostly from the Southern Hemisphere. The smaller subset of data from Northern Hemisphere (407) has a slope of $0.046^{\circ}$ cusp latitude per $1^{\circ}$ dipole tile angle, so they conclude that Northern and Southern Hemispheres act similarly.

Indeed, the Cluster satellites provide a good opportunity to research the location of mid-altitude cusp region ( $\left.4-8 R_{\mathrm{E}}\right)$ in both hemispheres. In this paper, we mainly focus on the inter-hemispheric comparison of cusp location dependence on dipole tilt angle. Our results suggest an inter-hemispheric asymmetry of the dipole tilt angle effect on the latitude location

\section{Data presentation}

\subsection{Identification criteria}

One way to discern the cusp region is by a diamagnetic depression (Tsyganenko and Russell, 1999). We can expect to see a decrease of the total magnetic field and some fluctua- tion in the magnetic field because of the diamagnetic effects of the incoming magnetosheath plasma and the weak background magnetic field in the mid-altitude cusp region. We can also see the magnetosheath-like (high density and low energy) plasma in this region. Based on these expectations our criteria to identify the cusp region are as follow: a decrease of the magnetic field strength from the background field greater than $1 \mathrm{nT}$ associated with some fluctuations; a sudden increase in the proton and electron density (greater than $5 \mathrm{~cm}^{-3}$ ); an electron thermal energy less than $100 \mathrm{eV}$; the presence of significant $\mathrm{He}^{++}$(greater than $0.5 \mathrm{~cm}^{-3}$ ), which signifies a solar wind origin. This method was also used by Zhou et al. (1999), who utilized the Polar satellite data in studying the cusp. As we know, the altitude of the cusp crossing for the Polar satellite is different from that for Cluster satellites, both in the Northern Hemisphere and the Southern Hemisphere. Nevertheless, we still use the broad criteria at the Polar altitude to identify the cusp region. Indeed, when we used the criteria to identify the cusp region in this study, we found the parameters varied in a consistent fashion according to the criteria used for the earlier study involving Polar, such that it is easy to identify the cusp region. This demonstrates that the criteria for the Polar crossings are also effective for the Cluster crossings. When we use these criteria to identify the cusp region in our study, we make use of the Cluster magnetic field, ion and electron data from the 
Flux Gate Magnetometer (FGM) (Balogh et al., 2001), Cluster Ion Spectrometer (CIS) (Rème et al., 2001) and Plasma Electron and Current Experiment (PEACE) (Johnstone et al., 1997), respectively.

Figure 1 shows an example of a cusp crossing by Cluster. The shadow indicates the cusp region based on the criteria. It extends from 16:19-16:32 UT on 10 August 2002, corresponding to 14.22-14.41 MLT. The upper trace shows that the residual of the magnitude of Cluster FGM data with the Tsyganeko 96 model (Tsyganenko and Stern, 1996). The second and the third traces show the PEACE electron density and energy. The highest electron density is $35 \mathrm{~cm}^{-3}$, and the highest electron energy is about $100 \mathrm{eV}$. The fourth trace shows the HIA $\mathrm{H}^{+}$density. The bottom traces show the $\mathrm{He}^{++}$density. The highest proton density is about $20 \mathrm{~cm}^{-3}$. These are all consistent with the criteria we have used above.

\subsection{Data set}

It is well known that the interplanetary magnetic field (IMF) plays a significant role in controlling the cusp location. For increasingly southward IMF, the cusp location is observed to move to lower latitudes, while for increasingly northward IMF, the cusp displays a slight tendency to move to higher latitudes (Newell and Meng, 1989). To limit the contribution of the IMF, we investigate the dipole tilt angle dependence on the cusp location only for northward IMF cases, for which the cusp is almost immune to the IMF variation (Newell and Meng, 1989; Palmroth et al., 2001; Pitout et al., 2006).

For each of the years 2001 through 2005, all the midaltitude cusp passes used here come from July to October. Then the Cluster spacecraft orbit has a good coverage at a wide magnetic local time (MLT) range (from 08:00 to $16: 00 \mathrm{MLT}$ ) in the dayside magnetosphere (Pitout et al., 2006).

Adding all these selection criteria together, we obtain a data set containing 95 polar cusp crossings (54 Northern Hemisphere crossings and 41 southern crossings) for northward IMF. The actual solar wind key parameter data for these events are available from the NASA's Space Physics Data Facility (SPDF).

\section{Data analysis}

Cluster, with a polar orbit, crosses both the northern and southern cusp regions each year. Since apogee lies slightly south of the equator, the southern crossings are sampled at slightly higher altitudes than the northern crossings and the local time coverage is also slightly different. Figure 2 depicts all the center position of the cusp crossings on a MLT vs. ILAT plot. Crossings occurring in the Northern and Southern Hemispheres are shown in Fig. 2a and b, respectively. Here, the MLT and ILAT are calculated by using the T96 model (Tsyganenko and Stern, 1996) in SM coordinates to trace the

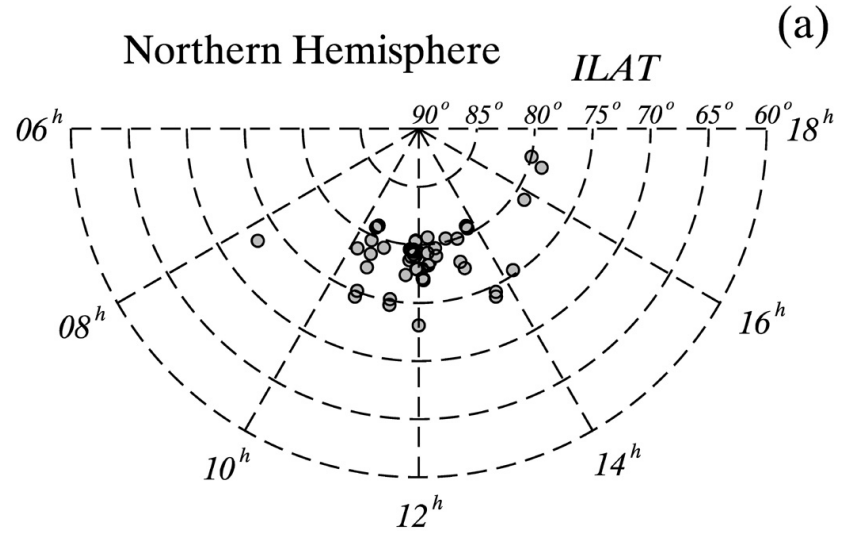

\section{$M L T$}

\section{Southern Hemisphere}

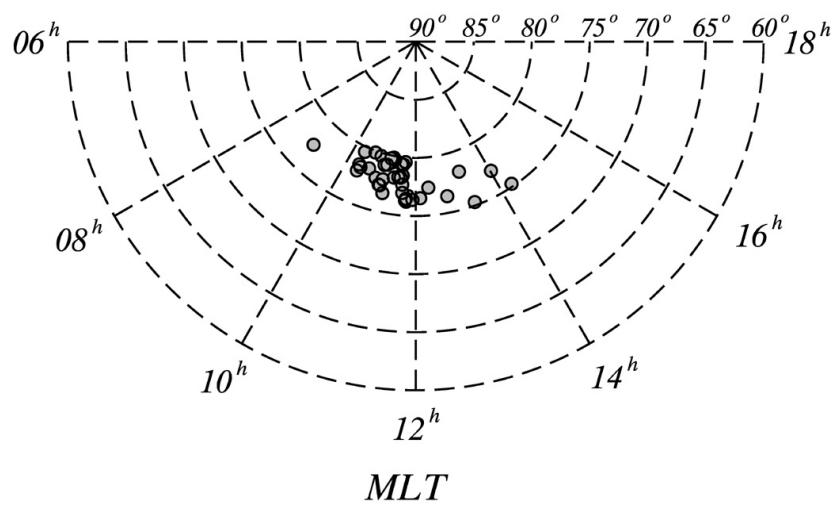

Fig. 2. Location of all the cusp crossings as a function of MLT and ILAT, (a) for the Northern Hemisphere, and (b) for the Southern Hemisphere.

magnetic field lines down to the surface of the Earth from each cusp position.

We note at this point that in the two most closely related studies to the present work, slightly different criteria were adopted. Zhou et al. (1999) chose to find ILAT values by mapping from the T89 model, in order to center the cusp crossings to the implied magnetic funnel from the field model. Pitout et al. did not use the mid cusp region to identify position, but used the poleward boundary in order to better reveal the effects of northward and southward $B_{\mathrm{z}}$. In the first case, the use of T89 shifts the effective ILAT values of each position higher, but we expect the dipole tilt dependence to be similar. In the second case, it is possible that the poleward boundary has a higher dependence on the dipole tilt since the cusp tends to widen during negative tilts, moving the poleward edge more poleward than the central cusp region.

At first glance in Fig. 2, most of the recorded cusps are located in a region extending from about 10:00 to 14:00 MLT and from about $75^{\circ}$ to $80^{\circ}$ ILAT, while the spatial distribution of northern cusp crossings seems more scattered than that of southern cusp crossings, and also it seems there is 

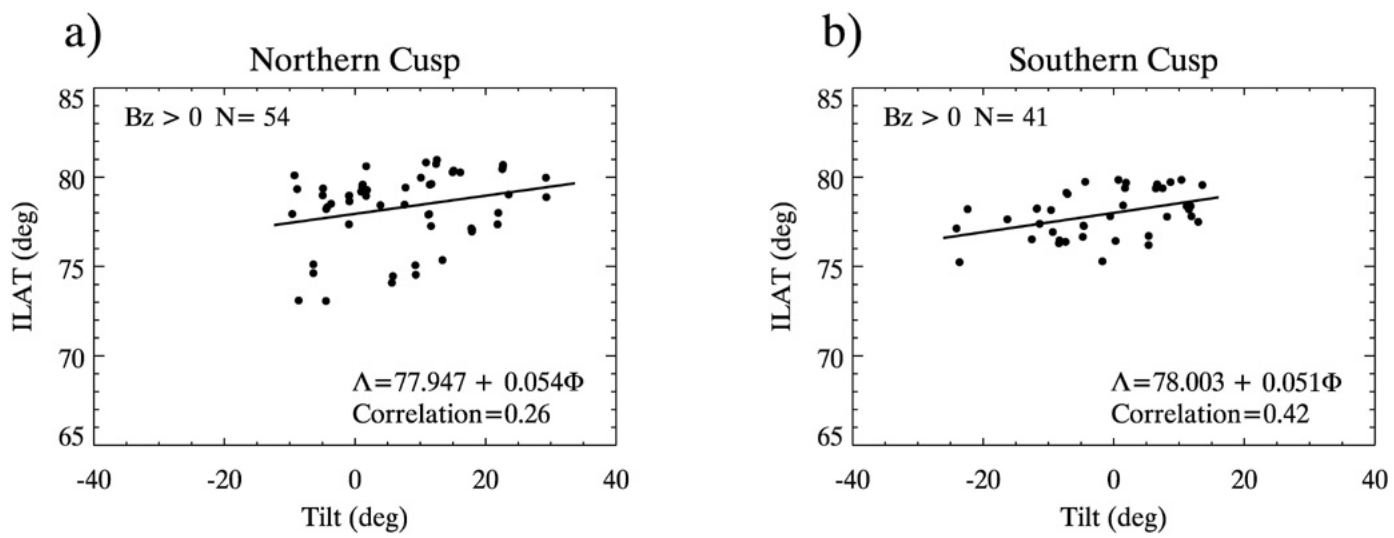

Fig. 3. The ILAT of the cusp location versus dipole tilt angle for cusp crossings corresponding to northward IMF, (a) for the northern cusp, (b) for the southern cusp.

an inter-hemisphere asymmetry. The center of the distribution for northern cusp crossings is located at 12:00 MLT and $78^{\circ}$ ILAT. For southern cusp crossings the center of distribution is located at 11:30 MLT and $78^{\circ}$ ILAT.

Figure 3 shows the ILAT of the center of the northern (Fig. 3a) and southern (Fig. 3b) cusp location as a function of the dipole tilt angle for cusp crossings corresponding to northward IMF. The dipole tilt angle is defined as the angle between the north dipole axis and the GSM Z-axis. This angle is positive when the dipole tilts toward the Sun, and negative when the dipole tilts away from the Sun. Here we just multiply the dipole tilt angle by -1 for the southern cusps. In Fig. 3 the dots show the cusp crossings according to their ILAT and dipole tilt angle. The linear fit of these dots in each panel in Fig. 3 gives the following relations:

$\Lambda_{\mathrm{NC}}=77.95+0.054 \Phi$

$\Lambda_{\mathrm{SC}}=78.00+0.051 \Phi$

Equation (1) is for the northern cusp and Eq. (2) is for the southern cusp. Here, $\Lambda_{\mathrm{NC}}$ and $\Lambda_{\mathrm{SC}}$ represent the ILAT of the northern and southern cusp center, respectively, and $\Phi$ is the dipole tilt angle. From Eqs. (1) and (2), we can see some properties for the cusp location in the Cluster orbit. The mean cusp position (for $0^{\circ}$ tilt angle) is $77.95^{\circ}$ ILAT in Northern Hemisphere and $78.00^{\circ}$ ILAT in Southern Hemisphere. The slope of the fit line is $0.054^{\circ}$ for the Northern Hemisphere and $0.051^{\circ}$ for the Southern Hemisphere, respectively. This means that the northern cusp moves $0.054^{\circ}$ ILAT for every $1^{\circ}$ increase in the dipole tilt angle and the southern cusp moves $0.051^{\circ}$ ILAT for every $1^{\circ}$ increase in the dipole tilt angle. The overall behavior of the cusp is the same (within the uncertainties) as that reported by some authors before (Newell and Meng, 1989; Zhou et al., 1999; Němeček et al., 2000), i.e. the slope is positive, which implies that the greater the dipole tilt angle $\Phi$ is, the higher the ILAT of the center of the cusp will be. However, previous authors have considered the Northern and Southern Hemispheres together to study the dependence of the cusp location on the dipole tilt angle. In our statistical study, the cusp crosses are divided into two groups, i.e. Northern Hemisphere and Southern Hemisphere (here we should note that the average altitudes are not the same in the Northern and Southern Hemisphere). The result shows that the cusp does not behave the same way for the two hemispheres, since different slopes are found from the linear fits. This result suggests an inter-hemispheric difference in the dependence of cusp latitudinal location on the dipole tilt angle. Figure 3a also shows a set of points with slightly lower ILAT values from the main distribution, but we have not attempted to separately analyze these.

\section{Discussion and conclusion}

As we mentioned above, some authors have studied the dependence of cusp locations on the dipole tilt with other different satellites observation at different altitudes. If we consider the previous results for different altitudes together, we can find that the higher the altitude of the satellite crossing, the larger the slope (Newell et al., 2006; Pitout et al., 2006; Zhou et al., 1999; Němeček et al., 2000).

Figure 4 summarizes the dipole angle tilt dependence of the cusp location in terms of the altitude. The slope observed in this study and other studies are represented by filled and open triangles (and lines indicating the range of altitudes), respectively. Point 1 is from Newell et al. (2006), point 2 from Pitout et al. (2006) (we should note that the point 2 in Fig. 4 is placed high, compared to the other studies, since they used the poleward boundary of the cusp, rather than the center of the cusp region, to identify position), point 3 (northern cusp only) from Zhou et al. (1999) and points 4 and 5 are from Němeček et al. (2000). The altitudes of the points in Fig. 4 are just the typical values. From Fig. 4, we can see that the 
slope increases monotonically with the increasing altitude. In Fig. 4 the points $\mathrm{c}$ and $\mathrm{d}$ indicate the results from our study for Northern and Southern Hemisphere at different average altitudes respectively, and do not follow the result from previous authors that the slope increases monotonically with the increasing altitude.

It should be also noted that the cusp crossings of the Cluster in this study are from 2001 to 2005. During this time period the line of apsides of the Cluster orbit changes, causing the apogee to occur progressively at higher altitudes in the Southern Hemisphere. Thus, the cusp crossings will occur at different altitudes in the two hemispheres from one year to the next. In this study, the mean altitude of the cusp crossings in Cluster obit was $5.2 R_{\mathrm{E}}$ in the Northern Hemisphere and 6.6 $R_{\mathrm{E}}$ in the Southern Hemisphere (the two mean altitudes are different in the Northern and Southern Hemisphere). Therefore, the slopes for the Northern and Southern Hemisphere are average values and the value for the Southern Hemisphere should be bigger than that for the Northern Hemisphere according to the trend in Fig. 4. According to this trend of the slope increasing with altitude, in Fig. 4 the point $d$ should have a value of about $0.069^{\circ}$, which is higher than its actual value of $0.051^{\circ}$; therefore, it gives a reversed result. Thus, our result in this study is contrary to the trend in Fig. 4 and suggests an inter-hemispheric asymmetry of the dipole tilt angle effects on the latitude location.

As mentioned above, for each of the years 2001 through 2005, all the Cluster cusp crosses in this study come from July to October (Pitout et al., 2006). This period is just in the summer and autumn for the Northern Hemisphere and the amount the solar EUV radiation reaching the ionosphere should be much different from that in the Southern Hemisphere (which is in the winter and spring). This will result in some different effect of the solar radiation on the two hemispheres.

Choe et al. (1973) predicted the dipole tilt angle effect by a simple magnetic dipole model plus magnetopause current. It has also been found that the dayside field-aligned currents (FACs) move poleward and equatorward in the summer and winter hemispheres, respectively (Fujii et al., 1981; Christiansen et al., 2002; Ohtani et al., 2005). That is consistent with the cusp location shift reported here. Newell and Meng (1989) supposed that the effect of the dipole tilt angle on the cusp location could be induced by the variation in the dayside current systems with changing dipole tilt angle. However, because the Cluster cusp crossings in this study are just in the summer and autumn for the Northern Hemisphere and the solar wind interact with Earth which keep an attitude of spin axis towards the sun, it may have some systematically different affected for the two hemispheres during this period (July to October) of each year. The ram pressure of the solar wind or the IMF orientation (i.e. $B_{\mathrm{y}}$ and $B_{\mathrm{x}}$ ) may have a different effect on the position of the cusp and on the dependency. These can be seen clearly in Figs. 2 and 3. In Fig. 2, the spatial distribution of northern cusp

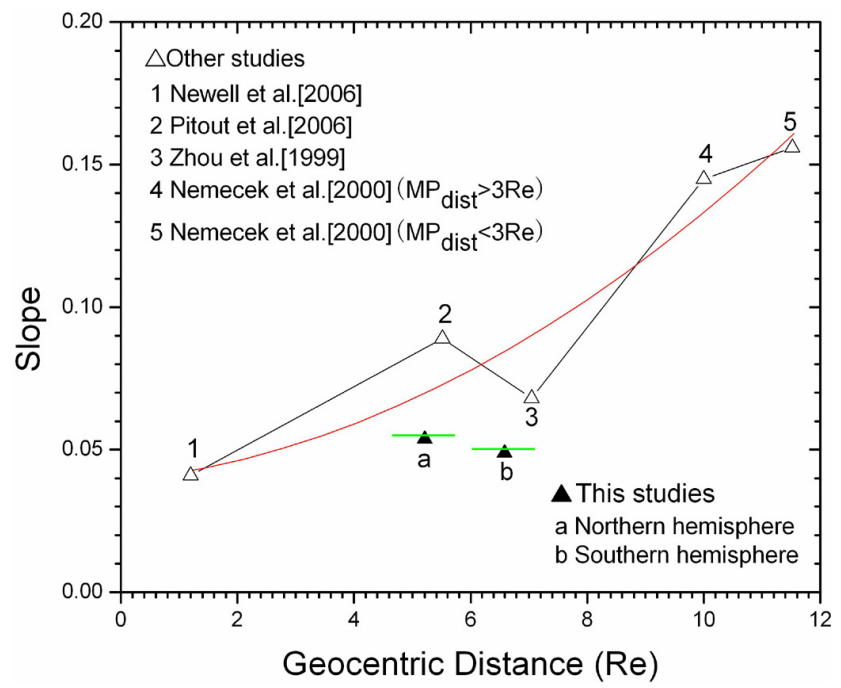

Fig. 4. The dependence of cusp location on the dipole tilt angle in different studies. Here we noted that point 2 should be lower because it corresponds to the poleward boundary of the cusp.

crossings seems more scattered than that of southern cusp crossings and it seems there is an inter-hemisphere asymmetry. In Fig. 3, the distribution of the northern cusp crossings clearly more scattered than that of the Southern Hemisphere. For the fit line for the two hemispheres, the standard deviation is $2.20^{\circ}$ in the Northern Hemisphere and $1.08^{\circ}$ in the Southern Hemisphere. Therefore, the difference of the two hemispheres may be resulted from the solar wind ram pressure or the IMF orientation. It seems reasonable to attribute the inter-hemispheric difference in the dipole tilt angle effect to the ram pressure of the solar wind or the IMF orientation (i.e. $B_{\mathrm{y}}$ and $B_{\mathrm{x}}$ ).

One plausible cause of the variation of the currents systems is the ionospheric conductivity changes (Fujii et al., 1981; Ohtani et al., 2005). The variation of the dipole tilt angle could control the amount of EUV radiation reaching the ionosphere and thus changing the amount and distribution of conductivity. But Christiansen et al. (2002) offered a different explanation. They argued that the seasonal dependence in the global FACs system is generated and maintained by various solar wind-magnetosphere interaction processes, such as quasi-viscous interaction and reconnection. This issue requires further investigations in the future.

\section{Summary}

In summary, we investigate the inter-hemispheric asymmetry of the location of the cusp and its dependence on the dipole tilt angle by analyzing the Cluster data obtained in the midaltitude cusp region at the altitude of $4-8 R_{\mathrm{E}}$. The results show the northern cusp moves $0.054^{\circ}$ ILAT for every $1^{\circ}$ increase in the dipole tilt angle at the mean altitude of $5.2 R_{\mathrm{E}}$, 
while the southern cusp moves $0.051^{\circ}$ ILAT for every $1^{\circ}$ increase in the dipole tilt angle at the mean altitude of $6.6 R_{\mathrm{E}}$. However, the previous observations of different satellites at different altitudes showed that the slope increases monotonically with the increasing altitude, i.e. the higher the altitude is; the larger the slope will be (this will be studied further in the other paper). The contrast between our results and the previous results, therefore, suggests an inter-hemispheric difference in the dependence of the cusp latitudinal location on the dipole tilt angle, which may reflect the inter-hemispheric asymmetry of the magnetospheric configuration, current system and solar wind interaction with magnetosphere between the Northern and Southern Hemispheres.

Acknowledgements. This research was supported by National Natural Science Foundation of China grant under 41074114, 40921063 and 41004076 , as well as by the Project Supported by the Specialized Research Fund for State Key Laboratories.

Topical Editor R. Nakamura thanks two anonymous referees for their help in evaluating this paper.

\section{References}

Balogh, A., Carr, C. M., Acuña, M. H., Dunlop, M. W., Beek, T. J., Brown, P., Fornacon, K.-H., Georgescu, E., Glassmeier, K.H., Harris, J., Musmann, G., Oddy, T., and Schwingenschuh, K.: The Cluster Magnetic Field Investigation: overview of in-flight performance and initial results, Ann. Geophys., 19, 1207-1217, doi:10.5194/angeo-19-1207-2001, 2001.

Burch, J. L.: Precipitation of low-energy electrons at high latitudes: Effects of interplanetary magnetic field and dipole tilt angle, J. Geophys. Res., 77, 6696-6707, 1972.

Choe, J. Y., Beard, D. B., and Sullivan, E. C.: Precise calculation of the magnetosphere surface for a tilted dipole, Planet Space Sci., 21, 485-498, 1973.

Christiansen F., Papitashvili, V. O., and Neubert, T.: Seasonal variations of high-latitude field-aligned currents inferred from Ørsted and Magsat observations, J. Geophys. Res., 107, 1029-1045, 2002.

Frank, L. A.: Plasma in the Earth's polar magnetosphere, J. Geophys. Res., 76, 5202-5219, 1971.

Fujii, R., Potemra, T. A., and Sugiura, M.: Seasonal dependence of large-scale Birkeland currents, Geophys. Res. Lett., 8, 11031106, 1981.

Heikkila, W. J. and Winingham, J. D.: Penetration of magnetosheath plasma to low altitudes through the dayside magnetospheric cusps, J. Geophys. Res., 76, 883-891, 1971.

Johnstone, A. D., Alsop, C., Burge, S., Carter, P. J., Coates, A. J., Coker, A. J., Fazakerley, A. N., Grande, M., Gowen, R. A., Gurgiolo, C., Hancock, B. K., Narheim, B., Preece. A., Sheather, P. H., Winningham, J. D., and Woodliffe, R. D.: Peace: A plasma electron and current experiment, Space Sci. Rev., 79, 351-398, doi:10.1023/A:1004938001388, 1997.
Němeček, Z., Merka, J., and Safrănkovă, J.: The tilt angle control of the outer cusp position, Geophys. Res. Lett., 27, 77-80, 2000.

Newell, P. T. and Meng, C.-I.: Dipole tilt angle effects on the latitude of the cusp and cleft/low-latitude boundary layer, J. Geophys. Res., 94, 6949-6953, 1989.

Newell, P. T., Sotirelis, T., and Liou, K.: Cusp latitude and the optimal solar wind coupling function, J. Geophys. Res., 111, A09207, doi:10.1029/2006JA011731, 2006.

Ohtani, S., Ueno, G., and Higuchi, T.: Annul and semiannual variations of the location and intensity of largescale field-aligned currents, J. Geophys. Res., 110, A01216, doi:10.1029/2004JA010634, 2005.

Palmroth, M., Laakso, H., and Pulkkinen, T.: Location of highaltitude cusp during steady solar wind conditions, J. Geophys. Res., 106, 21109-21122, 2001.

Pitout, F., Escoubet, C. P., Klecker, B., and Rème, H.: Cluster survey of the mid-altitude cusp: 1. size, location, and dynamics, Ann. Geophys., 24, 3011-3026, doi:10.5194/angeo-243011-2006, 2006.

Rème, H., Aoustin, C., Bosqued, J. M., Dandouras, I., Lavraud, B., Sauvaud, J. A., Barthe, A., Bouyssou, J., Camus, Th., CoeurJoly, O., Cros, A., Cuvilo, J., Ducay, F., Garbarowitz, Y., Medale, J. L., Penou, E., Perrier, H., Romefort, D., Rouzaud, J., Vallat, C., Alcaydé, D., Jacquey, C., Mazelle, C., d'Uston, C., Möbius, E., Kistler, L. M., Crocker, K., Granoff, M., Mouikis, C., Popecki, M., Vosbury, M., Klecker, B., Hovestadt, D., Kucharek, H., Kuenneth, E., Paschmann, G., Scholer, M., Sckopke, N., Seidenschwang, E., Carlson, C. W., Curtis, D. W., Ingraham, C., Lin, R. P., McFadden, J. P., Parks, G. K., Phan, T., Formisano, V., Amata, E., Bavassano-Cattaneo, M. B., Baldetti, P., Bruno, R., Chionchio, G., Di Lellis, A., Marcucci, M. F., Pallocchia, G., Korth, A., Daly, P. W., Graeve, B., Rosenbauer, H., Vasyliunas, V., McCarthy, M., Wilber, M., Eliasson, L., Lundin, R., Olsen, S., Shelley, E. G., Fuselier, S., Ghielmetti, A. G., Lennartsson, W., Escoubet, C. P., Balsiger, H., Friedel, R., Cao, J.-B., Kovrazhkin, R. A., Papamastorakis, I., Pellat, R., Scudder, J., and Sonnerup, B.: First multispacecraft ion measurements in and near the Earth's magnetosphere with the identical Cluster ion spectrometry (CIS) experiment, Ann. Geophys., 19, 1303-1354, doi:10.5194/angeo19-1303-2001, 2001.

Tsyganenko, N. A. and Stern, D. P.: Modeling the global magnetic field of the large-scale Birkeland current System, J. Geophys. Res., 101, 27187-27198, 1996.

Tsyganenko, N. A. and Russell, C. T.: Magnetic signatures of the distant polar cusps: Observations by Polar and quantitative modeling, J. Geophys. Res., 104, 24939-24955, 1999.

Zhou, X.-W., Russell, C. T., Le, G., Fuselier, S. A., and Scudder, J. D.: The Polar Cusp Location and its dependence on Dipole Tilt, Geophys. Res. Lett., 26, 429-432, 1999. 